\title{
Comparison of Target Volumes in Hepatocellular Carcinoma Delineated using Standard Method and Bolus Tracking Method
}

\author{
Debnarayan Dutta*, Ram Madhavan, Rupa Das, Rajesh Kannan, Anila K Raghu and Raghavandra \\ Holla \\ Department of Radiation Oncology, India
}

*Corresponding author: Debnarayan Dutta, Department of Head - Radiation Oncology, Amrita Institute of Medical Science, Ponekkara, 682026, Kochi, India

\begin{tabular}{|c|c|}
\hline ARTICLE INFO & ABSTRACT \\
\hline Received: 蔧 May 20, 2019 & Citation: Debnarayan D, Ram Madhavan, Rupa D, Rajesh Kannan, Anila K R, Raghavandra \\
\hline Published: 幽 May 28, 2019 & $\begin{array}{l}\text { H. Comparison of Target Volumes in Hepatocellular Carcinoma Delineated using Stand- } \\
\text { ard Method and Bolus Tracking Method. Biomed J Sci \& Tech Res 18(3)-2019. BJSTR. } \\
\text { MS.ID.003141. }\end{array}$ \\
\hline
\end{tabular}

\section{Editorial}

'Target delineation in radiation oncology is the most important step of treatment [1]. Usually, contrast CT scan is done to delineate the target. Target volume may be different at different phases of scan after contrast. The differential volume in different phases after contrast infusion (Arterial, Veinous, Portal phase) may be responsible for either increase or decrease or even miss a part of the tumour which may have impact on clinical outcome. After contrast infusion, there is a time lag for the appropriate contrast material to reach the tumour tissue before it is washed out through the veins [2]. Appropriate timing of the scan is critical for optimal image quality, enhancement of the lesion and appropriate delineation of the target [3]. In 'standard' contrast CT scan, there is a 'fixed' time interval between the infusion and imaging scan. However, the 'time lag' or the time required to reach the tumour or target depends upon various patient related factors, such as
a. Blood Pressure,
b. Heart rate,
c. Status of cardiac contractility (ejection fraction),
d. Patient position and
e. Height of the patient.

There is a need to individualize the 'lag time' between the infusion of contrast and scan. 'Bolus tracking' is a contrast CT scan method which is individualized to improve the image quality and hence delineation of the target [4]. In the present study, 'bolus tracking' was evaluated for primary liver tumours. In primary liver tumour, appropriate contrast imaging is critical to delineate the target. Contrast enhancing lesion volume in arterial, venous and portal phase may differ with timing of imaging after contrast infusion, and the target volume will be different or even miss the actual target in inappropriately timed scan. 'Bolus tracking' may help for appropriate phase imaging and proper delineation of the target. Breathing pattern and imaging in realtion to breathing cycle (eg- end of expiration) may also influence target volume. The bolus tracking method and standard CT simulation method work-flow is mentioned in Figure 1.

Standard technique: During CT simulation for SBRT for primary liver tumour (Hepatocellular carcinoma), usually a contrast CT scan is taken for target volume delineation. An $80 \mathrm{~mL}$ of contrast is given using $20 \mathrm{G}$ cannula with a flow rate of 2.5 to $3 \mathrm{~mL}$ per second. The scans are taken after fixed arbitrary time delays, arterial phase is taken 2 seconds, portal phase 12 seconds and venous phase 15 seconds delay time after contrast injection. Images taken in different phases are used for target delineation. Bolus tracking during multiphase contrast CT scan is often used in diagnostic radiology, mostly for liver tumours to enhance the visibility of tumours. However, in planning CT simulation, bolus tracking 
method is not usually applied. In high dose per fraction short course treatment (stereotactic radiosurgery) for primary liver tumours we considered triphasic CT simulation using 'bolus' tracking for appropriate delineation of target volume. Bolus tracking technique: CT scans were acquired using 4D CT simulator GE OPTIMA 580WRT with 16 slice large bore size of $80 \mathrm{~cm}$ and flat couch [5].

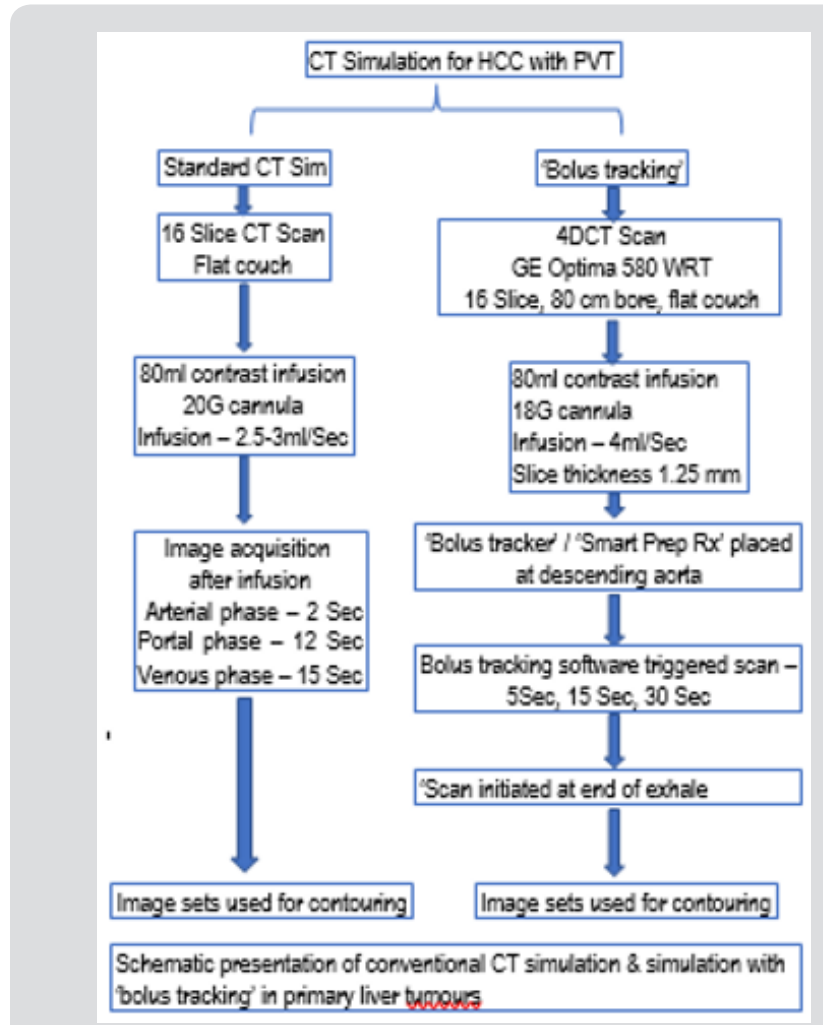

Figure 1: Schematic presentation of conventional CT simulation \& simulation with 'bolus tracking' in primary liver tumours.

Initially $80 \mathrm{~mL}$ of contrast is injected using $18 \mathrm{G}$ cannula at a flow rate of $4 \mathrm{~mL}$ per second. The slice thickness used is $1.25 \mathrm{~mm}$. 'Bolus' tracker or Smart Prep RX is placed at descending aorta (Figure 2). Bolus tracking software trigerred scans of liver were taken at 5 seconds, 15 seconds and 30 seconds following aortic enhancement corresponding to aortic, portal and venous enhancement phases of liver (Figure 3). These scans were initiated at the end of exhale. Arterial phase was initiated (triggered) when the contrast reaches the bolus tracker. Triggering of arterial phase was done by bolus tracker and predifined Houndsfield unit. Veinous phase and portal phase was initiated at $15 \mathrm{sec}$ and $30 \mathrm{sec}$ after triggering of the scan. Bolus tracking mainly help in appropriate time (when the contrast reaches the bolus tracking region, defined by Hounds field unit change) for triggering the scan. Bolus tracking was well tolerated by our patients. The tumor enhancement and portal vein thrombus was appropriately visualized with bolus tracking.

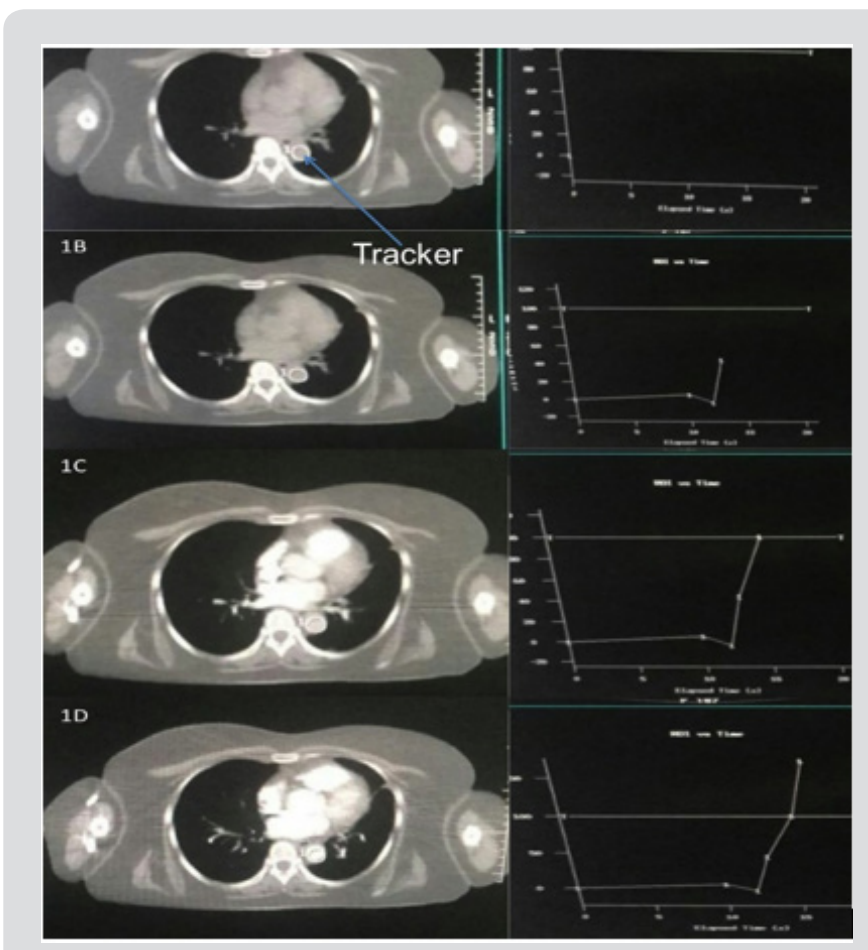

Figure 2: Pictorial presentation of standard simulation and bolus tracking simulation method.

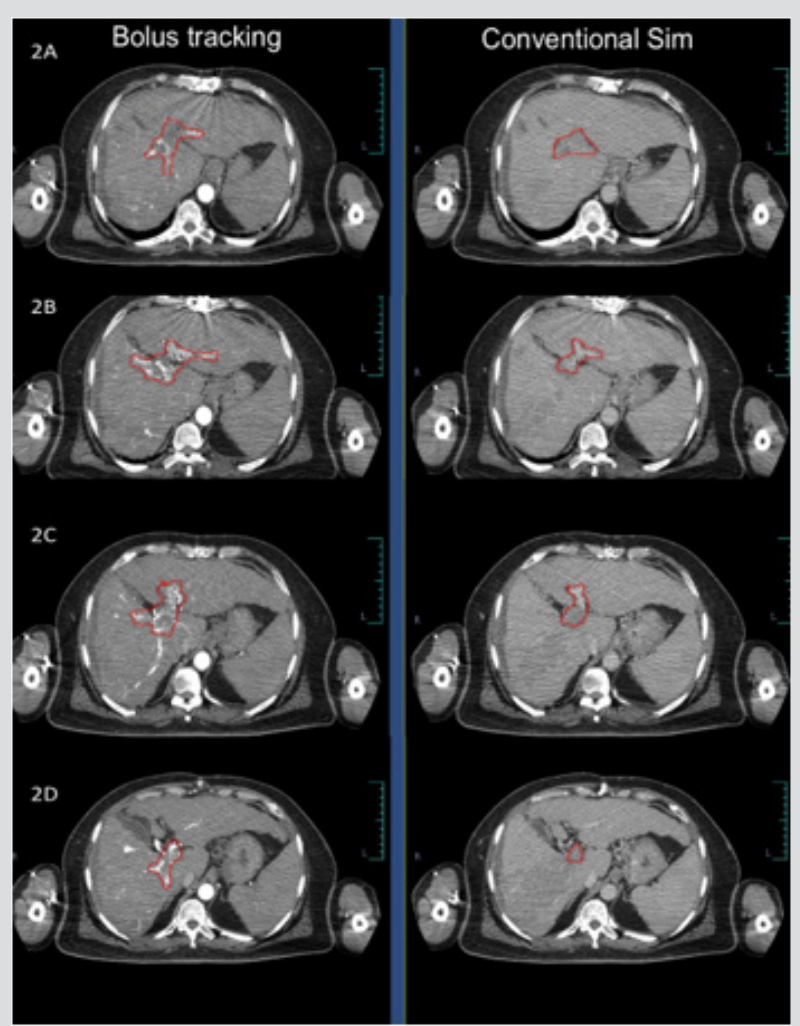

Figure 3: Comparison of target visualization with and without bolus tracking. 
Target volume was better defined in arterial and portal phases after bolus tracking when compared to non-bolus tracked conventional contrast CT simulation scans (Figure 3). Imaging of liver is always facinating. Even in early CT scan era there was a concept of using contrast in phased manner to improve the contrast between liver parenchyma and tumour tissue [6,7]. Different dynamic scans use differential time gap between contrast infusion and scanning. Apart from the differential time gap, contrast flow rate and amount of contrast influence the image quality. Radiological diagnosis of the lesions were done mostly by the enhancement pattern in different phases and the architecture of the lesion. 'Multiphasic' CT scan for diagnosis of liver tumours are in use for last few decades. Multiphasic CT scan utilise the differential time gap between contrast infusion and image acquisition in arterial, venous or portal phase. Hepatic lesions are better visible in specific sequences, such as liver metastasis in arterial phase, primary liver tumour in venous phase and portal vein thrmobosis in portal phase. Liver parenchyma also uptake contrast. Differential contrast uptake between normal liver parenchyma and tumour defines the target volume.

Appropriately timed image acquisition after contrast infusion helps in defining the tumour appropriately. Bolus tracking helps in triggering of image acquisition at appropirate time and hence helps in appropriate deliniation of target. Bolus tracking nullifies the patient related factors (ejection fraction, heart rate, blood pressure etc) influencing the flow rate of blood and contrast. Bolus tracking need 4D CT scan with 'Bolus tracker' or 'Smart PreRX' software to track the bolus, trigger the scan when the bolus (contrast) reaches the descending aorta and is not routinely used for radiation therapy planning in liver tumours. In radiation oncology, contouring done in contrast CT scan. Imaging at different phase in different tumours will provide appropriate anatomical information. 'Bolus tracking' with appropriate imaging will provide us appropriate phase information and contouring of the target. Bolus tracking have shown significant concordance in appropriate tumour volume delineation in pancreatic tumour [8]. In stereotactic radiosurgery, appropriate delineation of target is critical as there is no clinical target volume (CTV) and set up margins (PTV) are minimal [1].
Bolus tracking will improve the accuracy of tumour volume delineation and may reduce geographical miss as well as marginal recurrences. In summary, radiation oncologists consistently preferred arterial phase for better target volume delineation and portal venous phase for delineation of tumor thrombus in portal vein. 'Bolus tracking' method of contrast CT scan for tumour delineation provides appropriate target definition. In our experience with thirty-six primary liver tumours treated with 'bolus tracking' method during CT simulation have given appropriate deliniation of target. Our first two patients where target volume was compared with non-bolus and bolus tracking methods as well, there was a definitive better visualization of target volume with bolus tracking. Hence, in our institute bolus tracking is considered for all primary liver tumours. From our institutional experience we recommend bolus tracking with arterial phase for hepato cellular tumor delineation and portal venous phase for portal vein tumor thrombus delineation.

\section{References}

1. Koay EJ, Hall W, Park PC, Erickson B, Herman JM (2018) The role of imaging in the clinical practice of radiation oncology for pancreatic cancer. Abdom Radiol (NY) 43(2): 393-403.

2. Dinkel HP, Fieger M, Knüpffer J, Moll R, Schindler G (1998) Optimizing liver contrast in helical liver CT: value of a real-time bolus-triggering technique. Eur Radiol 8(9): 1608-1612.

3. Marchal G, Baert AL (1992) Dynamic CT of the liver. Radiologe 32(5): 211-216.

4. Shimizu T, Misaki T, Yamamoto K, Sueyoshi K, Narabayashi I (2000) Helical CT of the liver with computer-assisted bolus-tracking technology: scan delay of arterial phase scanning and effect of flow rates. J Comput Assist Tomogr 24(2): 219-23.

5. GE Healthcare, Ti P Training in Partnership Smart Prep Quick Steps.

6. Ichikawa T, Ohtomo K, Takahashi S (1996) Hepatocellular carcinoma: detection with double-phase helical CT during arterial portography. Radiology 198(1): 284-287.

7. Baron RL (1994) Understanding and optimizing use of contrast material for CT of the liver. AJR Am J Roentgenol 163(2): 323-331.

8. Godfrey DJ, Patel BN, Adamson JD, Subashi E, Salama JK, Palta M (2017) Triphasic contrast enhanced CT simulation with bolus tracking for pancreas SBRT target delineation. Pract Radiat Oncol (6): e489-e497.

\section{ISSN: 2574-1241}

DOI: 10.26717/BJSTR.2019.18.003141

Debnarayan Dutta. Biomed J Sci \& Tech Res

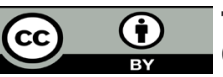

This work is licensed under Creative Commons Attribution 4.0 License

Submission Link: https://biomedres.us/submit-manuscript.php

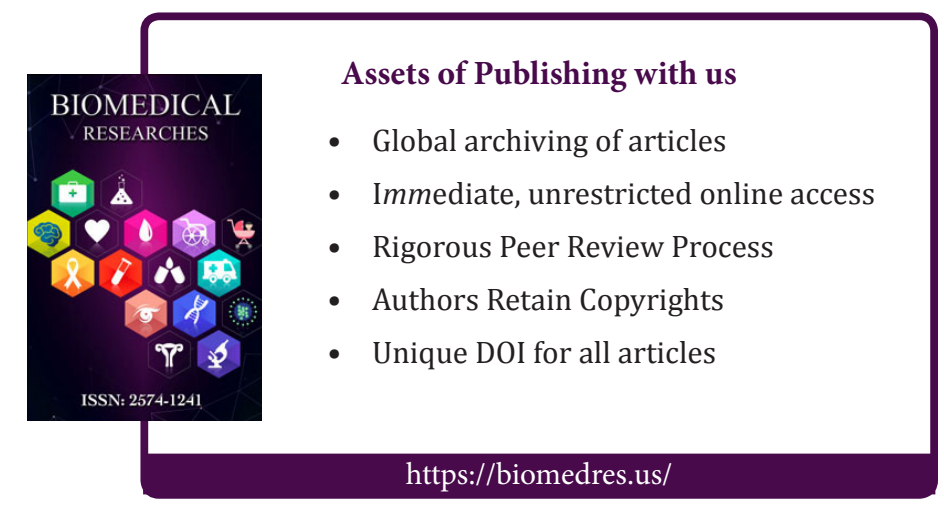

